\title{
Repeated isoflurane exposures of neonatal rats contribute to cognitive dysfunction in juvenile animals: the role of miR-497 in isoflurane-induced neurotoxicity
}

\author{
Yuanyuan Que, Fangxiang Zhang, Jing Peng, Zhuhua Zhang, Duwen Zhang, Minghai He
}

Department of Anesthesiology, Guizhou Provincial People's Hospital, China

\begin{abstract}
Introduction. Isoflurane anesthesia at the period of brain development can lead to neurotoxicity and long-term cognitive impairment. This study aimed to investigate the role of miR-497 on isoflurane-induced neurotoxicity. Material and methods. Neonatal rats (P7) were subject to isoflurane for $2 \mathrm{~h}$ at P7, P9, and P11. MiR-497 and neuron apoptosis were evaluated in hippocampal tissue by qRT-PCR and western blot. Fear conditioning test and Morris water maze were performed to determine cognitive function. The cell viability of isolated hippocampal neuronal cells exposed to isoflurane was measured using MTT test. The regulation of phospholipase D1 (PLD1) by miR-497 in isolated hippocampal neuronal cells was evaluated by luciferase reporter assays and western blot. Immunohistochemistry and TUNEL staining were employed to examine the PLD1 expression and neuronal cell apoptosis in hippocampus of neonatal rats, respectively.

Results. Repeated isoflurane anesthesia led to neurons' apoptosis and long-term cognitive impairment. Isoflurane exposure led to apoptosis and viability reduction in hippocampal neuronal cells. MiR-497 was observed to be upregulated after isoflurane exposure both in vivo and in vitro. Knockdown of miR-497 attenuated isoflurane-induced neuronal cells apoptosis and viability reduction. Furthermore, PLD1 was predicted and then validated as a novel target of miR-497. miR-497 could negatively regulate PLD1 by binding to its 3'-untranslated region. Downregulation of PLD1 was also observed after isoflurane exposure in neonatal rat hippocampus and hippocampal primary neuronal cell cultures.

Conclusions. Induction of miR-497 was involved in isoflurane anesthesia-induced cognitive impairment and neuronal cell apoptosis by targeting PLD1. miR-497 may be a novel potential mechanism in isoflurane-induced neurotoxicity so that our findings provide new insight into a better and understanding of the clinical application of isoflurane. (Folia Histochemica et Cytobiologica 2021, Vol. 59, No. 2, 114-123)
\end{abstract}

Key words: isoflurane; neurotoxicity; rat pups; hippocampal neurons; miR-497; phospholipase D1; cognitive impairment; qPCR; western blot

\section{Introduction}

Early exposure to anesthetic agents is deleterious to nervous system development, impairs learning and

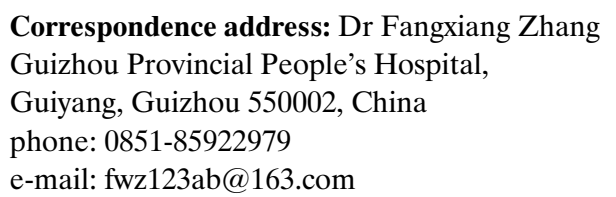

memory, this neurocognitive dysfunction will even persist into later adulthood [1]. As millions of young children are exposed to anesthesia every year for various reasons, and there is raising concern about risks that may result from anesthesia exposure. Studies have shown that children who received multiple anesthesias are likely to develop a learning disability $[2,3]$. Animal studies have found that both intravenous and inhalation anesthetic agents can cause central nerve cell apoptosis, especially during the critical period of

This article is available in open access under Creative Common Attribution-Non-Commercial-No Derivatives 4.0 International (CC BY-NC-ND 4.0) license, allowing to download articles and share them with others as long as they credit the authors and the publisher, but without permission to change them in any way or use them commercially. (C)Polish Society for Histochemistry and Cytochemistry 
brain development [4-6]. It was demonstrated that in postnatal day 7 (P7) inhalation of anesthetic isoflurane by rat pups increased neuronal cell apoptosis, especially in thalamic nuclei [7], and inhibited the proliferation of neural progenitor cells in hippocampus, which ultimately led to poor learning and memory in P7 rats; however, the same administration of isoflurane to P60 rats seemed to play an opposite role [1]. Although a rising number of studies have focused on anesthesia-induced detrimental effects on cognitive function [8-10], it is still insufficient to understand the underlying mechanisms.

MicroRNAs (miRNAs) are small non-coding RNAs that complementarily bind to the 3'-untranslated region (3'-UTR) of targets to regulate their expression [11]. The highly multitudinous miRNAs have been involved in the regulation of neurodevelopment, neuronal inflammation, neuronal degeneration and apoptosis, cognition, memory, and other processes $[12,13]$. For example, Mai et al. showed that intranasal injection of miR-146a can reduce the cognitive dysfunction in a murine Alzheimer's disease model, and that miR-146a exerts an inhibitory effect on amyloid $\beta(\mathrm{A} \beta)$ precipitation, tau protein phosphorylation, and neuroinflammation in the hippocampus [14]. Some recent studies have found that the use of anesthetics has an effect on the expression of miRNAs in the brain, and more in-depth studies have shown a link between the expression of miRNAs and cognitive impairment associated with anesthesia [15-18].

The highly conserved miR-195/497 cluster is located on human chromosome 17p13.1 [19]. Yin et al. found an increase of miR-497 expression in the brain tissue of transient middle cerebral artery occlusion (MCAO) mice. Further studies found that the knocking down of miR-497 can reduce the neuronal apoptosis caused by oxygen and glucose deprivation [20]. Another study demonstrated that the expression of miR-497 in neuroblastoma cell exposed to ethanol was significantly increased, and miR-497 was subsequently proved to play an important regulatory role in the nerve cells apoptosis induced by ethanol by targeting anti-apoptosis protein BCL2 [21]. These studies indicated that miR-497 may play an important role in neuronal apoptosis.

In this study, we used neonatal rats and primary neuronal cell cultures exposed to isoflurane to detect the expression of miR-497, and the effects of miR-497 on neurotoxicity induced by isoflurane was investigated by downregulating the expression of miR-497. The present study aimed to investigate the potential mechanism underlying the effect of miR-497 on learning and memory impairment in isoflurane-treated rats and may provide a theoretical basis for the molecular mechanism of isoflurane in the clinical treatment of neuronal damage.

\section{Materials and methods}

Experimental procedures were carried out in strict accordance with the recommendations in the Guide for the Care and Use of Laboratory Animals of the Guizhou Provincial People's Hospital and the protocol was approved by the Hospital.

Animals. Seven-day-old (P7) Sprague-Dawley male rat pups were randomly assigned to two groups: the control group $(n=20)$ and isoflurane group $(n=20)$. Rats in the isoflurane group were exposed for $2 \mathrm{~h}$ to $2 \%$ isoflurane in $25 \%$ oxygen in a temperature-controlled chamber, and the isoflurane exposure was conducted on postnatal $(\mathrm{P})$ days $\mathrm{P} 7, \mathrm{P} 9$, and $\mathrm{P} 11$. The time and concentration of isoflurane exposure was selected according to previous research with some modifications [22,23]. Rats in the control group received control gas ( $25 \%$ oxygen) in a similar chamber for $2 \mathrm{~h}$. The chamber was kept in a homeothermic incubator to maintain the temperature at $37^{\circ} \mathrm{C}$. After $24 \mathrm{~h}$ of the last exposure, half of the rat pups from each group were euthanized by intraperitoneal injection of pentobarbital sodium (150 mg/kg; Sigma-Aldrich, St. Louis, MO, USA) overdose, the brains were dissected, and the hippocampus of each brain was immediately frozen in liquid nitrogen and stored at $-80^{\circ} \mathrm{C}$ for western blot and qRT-PCR analysis. The remaining animals were used for a behavioral study to determine their cognitive function after 7 weeks (at P57).

TUNEL staining and immunohistochemistry. The dissected hippocampus was immediately immersed in $4 \%$ formaldehyde at $4^{\circ} \mathrm{C}$ for $24 \mathrm{~h}$ and then embedded in paraffin. The embedded tissue was then sectioned into $5 \mu \mathrm{m}$ slides. As for TUNEL staining, a commercial TUNEL Apoptosis Detection Kit (Millipore, Billerica, MA, USA) was employed according to the manufacturer's instruction. Briefly, the sections were deparaffinized and dehydrated using xylene and ethanol. After rehydration, the sections were rinsed with phosphate-buffered saline (PBS) and incubated with proteinase $\mathrm{K}$, followed by incubation with TUNEL reaction mixture for $1 \mathrm{~h}$ at $37^{\circ} \mathrm{C}$, then further stained using DAPI. The sections were examined under a laser scanning confocal microscope LSM710 (Carl Zeiss, Jena, Germany).

For immunohistochemistry, the paraffin-embedded sections were deparaffinized and dehydrated as described above, and then incubated with anti-PLD1 antibody (sc28314, 1:50, Santa Cruz Biotechnology, Santa Cruz, CA, USA) at $4^{\circ} \mathrm{C}$ for $12 \mathrm{~h}$. After washing, the sections were incubated with HRP-conjugated secondary antibody for $1.5 \mathrm{~h}$ at room temperature (RT) and then stained with diaminobenzidine tetrachloride (DAB) and counterstained with hematoxylin. 
Isolation and primary cultures of hippocampal neurons. The brains of Sprague-Dawley rat embryos (E18) were dissected, and primary cultures were isolated as reported previously [24]. Briefly, the hippocampus was dissected from the brain, followed by trituration dissociation and enzymatic digestion ( $0.3 \%$ trypsin), and seeded in poly-D-lysine-precoated 6-well plates (Corning, NY, USA). Cultures were maintained in a humidified incubator with $5 \% \mathrm{CO}_{2}$ at $37^{\circ} \mathrm{C}$ in Neurobasal medium (Gibco, Frederick, MD, USA) with $2 \mathrm{mM}$ glutamine, $2 \%$ B27, 100 units $/ \mathrm{mL}$ penicillin, and $100 \mathrm{mg} / \mathrm{mL}$ streptomycin. The density of neurons in plates was $10^{6} \mathrm{cells} / \mathrm{cm}^{2}$. Treatments were performed on the $6^{\text {th }}$ day in vitro.

Cell viability assays. Primary neurons were exposed to normal air (control group), 2\% (ISO-2 group), or 5\% (ISO-5 group) isoflurane for the indicated time. The cell viability was measured using 3-(4, 5-dimethylthiazol-2-yl)-2, 5-diphenyltetrazolium bromide (MTT) (Sigma-Aldrich, Taufkirchen, Germany). After $24 \mathrm{~h}$ of the exposure to the indicated doses of isoflurane, primary neuronal cells were cultured at normal air for 1, 3 and 7 days, cell viability was determined by incubation with $25 \mu 10.5 \mathrm{mg} / \mathrm{mL}$ of MTT reagent at $37^{\circ} \mathrm{C}$ for $4 \mathrm{~h}$, followed by the addition of $150 \mu \mathrm{l}$ dimethyl sulphoxide (DMSO) to resolve the generated formazan. The absorbance at a wavelength of $570 \mathrm{~nm}$ was measured using a microplate reader. Data were collected from 3 independent experiments.

RNA extraction and quantitative real-time PCR (qRT-PCR). Total RNA was isolated from hippocampal tissue samples and neurons cell by Trizol reagent (Invitrogen, Grand Island, NY, USA). RNAs were reverse transcribed into cDNA using miScript Reverse Transcription Kit (QIAGEN, Dusseldorf, Germany) following the manufacturer's protocol. qRT-PCR was performed with SYBR Premix EX Taq TM II (Takara, Dalian, China) on the real time PCR detection system ABI7900 (Thermo Fisher Scientific, Waltham, MA, USA). GAPDH or U6 was used as the internal reference, and relative expression was calculated by $2^{-\Delta \Delta} \mathrm{Ct}$ method. The primer sequences were as follows: miR-497-F: GTCGTATCCAGTGCAGGGTCCGAGGT; R: ATTCGCACTGGATACGACTACAAACC; GAPDH-F: GTGAACCATGAGAAGTATGACAAC; R: CATGAGTCCTTCCACGATACC; U6-F: GCTTCGGCAGCACATATACTAAAAT; R: CGCTTCACGAATTTGCGTGTCAT.

Luciferase assay. The 3'-UTR of phospholipase D1 (PLD1) harboring the seed sequence of the miR-497 wild-type binding sites or mutant binding sites was inserted into the pmirGLO dual-luciferase vector (pmirGLO Vector) containing firefly and renilla luciferase genes (Promega, Madison, WI, USA). The wild-type or the mutant dual-luciferase vectors were co-transfected with $20 \mathrm{nM}$ miR-497 mimic or inhibitor or their negative control into $293 \mathrm{~T}$ cells using Lipofectamine 2000 (Invitrogen) according to the manufacturer's instructions. After $48 \mathrm{~h}$ of transfection, cells were harvested, and luciferase activity was detected using the Dual-Luciferase Assay System (Promega). The ratio of firefly activity to renilla activity was defined as the relative reporter activity. All experiments were performed in triplicate.

Transfection of neurons. The miR-497 mimic or inhibitor or their negative control was transfected into the primary neuron using Lipofectamine 2000 (Invitrogen) according to the manufacturer's instructions. The final concentration of small RNAs was $50 \mathrm{nM}$.

Western blot analysis. Ground tissue or neurons were lysed with RIPA lysis buffer, and the protein concentration was quantified using BCA protein assay kit (Beyotime, Shanghai, China). Following the extraction of total proteins, $20 \mu \mathrm{g}$ of proteins were loaded and separated on $10 \%$ sodium dodecyl sulfate polyacrylamide gels. The separated proteins were transferred onto polyvinylidene fluoride membrane (Millipore). After blocking with nonfat dry milk $(2.5 \%)$, the membranes were incubated with primary antibodies overnight at $4^{\circ} \mathrm{C}$. The next day, membranes washed with TBST for every ten minutes were incubated with secondary antibodies conjugated with Horse Radish Peroxidase (HRP) for $1 \mathrm{~h}$ at RT. Antibodies used were as followed: anti-cleaved caspase 3 (ab2302, 1:1000, Abcam, Cambridge, MA, USA), anti- $\beta$-actin (ab8226, 1:1000, Abcam), anti-PLD1 (ab50695, 1:1000, Abcam). The membranes were subsequently incubated with HRP-conjugated secondary antibodies and detected with Pierce ${ }^{\mathrm{TM}}$ ECL Plus Western Blotting Substrate (32134, Thermo Fisher Scientific). The images were captured with SmartView Pro 2000 (UVCI-2100, Major Science, Saratoga, CA, USA). The bands were analyzed using Image J (version 1.8.0_112, National Institutes of Health, Bethesda, MD, USA).

Morris water maze. To assess the learning and memory functions of juvenile rats, animals from all the groups were subjected to the Morris water maze test at P60. A platform located at a fixed position in a circular water pool (100 cm in diameter, $80 \mathrm{~cm}$ in height) was submerged $2 \mathrm{~cm}$ below the water surface. The water temperature was set at $23^{\circ} \mathrm{C}$. The training was conducted twice per day for seven consecutive days. During the trials, the swimming animals were forced to find the hidden platform within a maximum of $60 \mathrm{~s}$. If the rat failed to find the platform within $60 \mathrm{~s}$, it would be manually guided to the platform and allowed to stay on it for $15 \mathrm{~s}$. The time spent on locating the platform (latency) was recorded and analyzed. The probe trials were performed on the $8^{\text {th }}$ day, the platform was removed, then, 


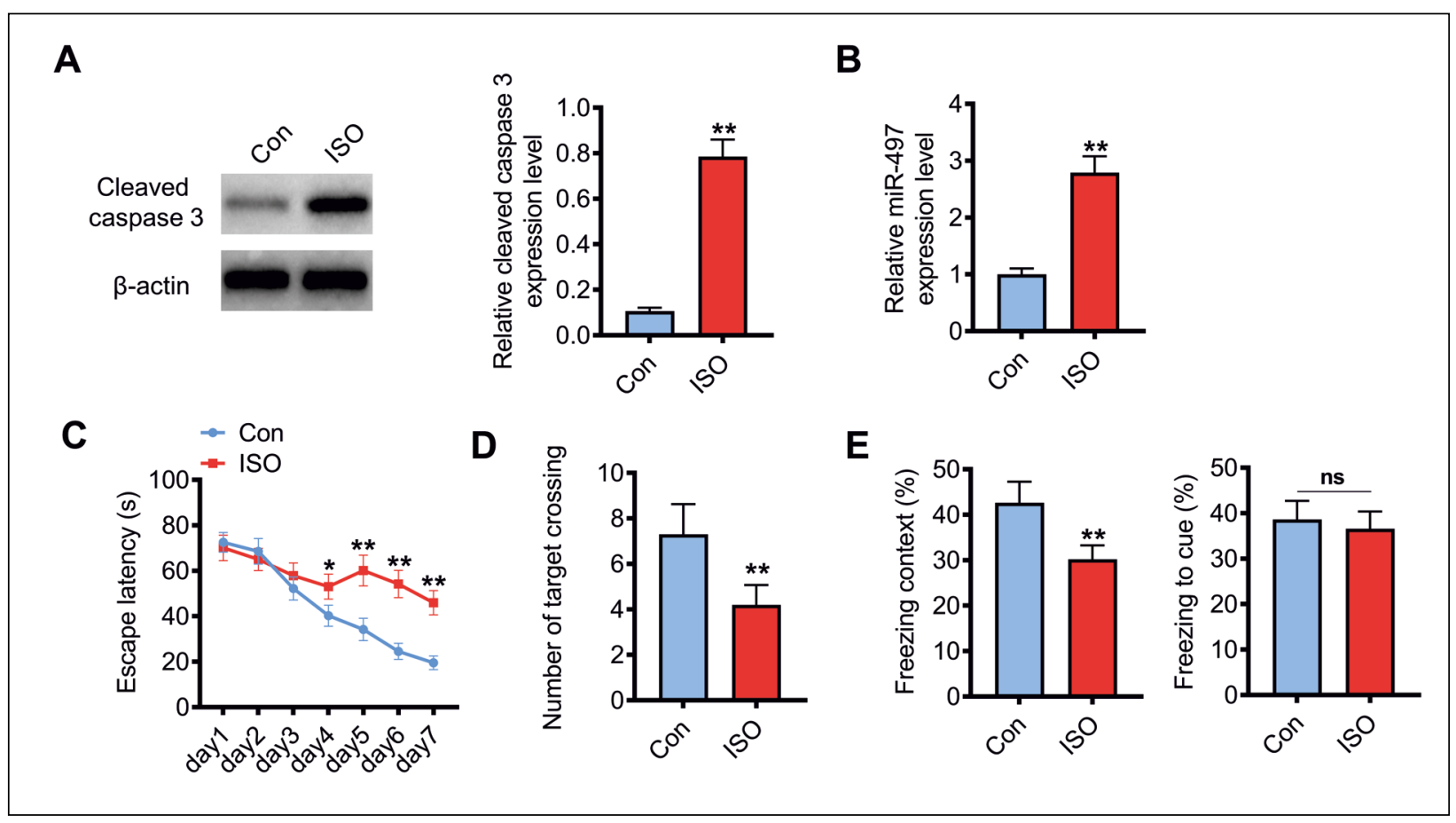

Figure 1. Isoflurane (ISO) anesthesia-induced neurotoxicity and miR-497 expression level in neonatal rat hippocampus. A. The expression of cleaved caspase 3 (western blot) and (B) miR-497 expression (RT-qPCR) in neonatal rat hippocampus after exposure of rat pups to ISO at the postnatal $(\mathrm{P})$ day 7, P9, and P11 (P7, P9, and P11) $(\mathrm{n}=10)$. C. Place trial demonstrating latency for rats to reach platform measuring spatial reference memory and learning as described in Methods. D. Probe trial demonstrating the target platform crossing times of juvenile rats in Morris water maze. E. The freezing time to context and the freezing time to cue in the fear conditioning test $(\mathrm{n}=10)$. Fear conditioning test was performed at P57, Morris water maze was performed at P60 on the same rat. ${ }^{*} p<0.05,{ }^{*} p<0.01$, compared with the control group.

the number of times the rats crossed the area where the platform was located in the target quadrant was recorded. The entire test was recorded and analyzed by an MS-type Water Maze Video analysis system (Chengdu Instrument Ltd., Chengdu, China).

Fear conditioning test. On P57, rats were subjected to fear conditioning test. Each animal was placed in a conditioning chamber with a stainless-steel shock grid floor. The rats were allowed to explore the chamber for $3 \mathrm{~min}$ for adaption, and then rats were given for $30 \mathrm{sec}$ tone-foot shock $(80 \mathrm{~dB}, 1 \mathrm{kHz})$ followed by a 2 -s foot shock $(1.0 \mathrm{~mA})$, this process was repeated 3 times with an intertribal interval of $60 \mathrm{~s}$. Then, the animal was removed from the test chamber $60 \mathrm{sec}$ after conditioning training. The contextual fear memory was measured $24 \mathrm{~h}$ after training for $5 \mathrm{~min}$ by placing the rat back in the original training chamber to monitor freezing behavior. The cued fear memory was tested $24 \mathrm{~h}$ after training, and the rats were placed in a novel chamber that had different context and smell with a continuous 3-min training tone presentation to monitor freezing behavior. Freezing behavior, defined as the absence of all visible movement of the body except for respiration, was recorded by a video camera with a high resolution on top of the chamber.
Statistical analysis. Statistical analyses were performed using SPSS 19.0 software (IBM SPSS Inc., Chicago, IL, USA) and Prism 7.0 software (GraphPad Software, La Jolla, CA, USA). Behavioral studies were analyzed with two-way ANOVA (treatment and time). Other data were analyzed using Student's $t$-test and one-way ANOVA. All the values were presented as the mean \pm SD. $P<0.05$ was considered statistically significant.

\section{Results}

\section{Expression of miR-497 was upregulated}

in hippocampus of neonatal rats induced by isoflurane After isoflurane exposure on P7, P9, and P11, the hippocampus of the neonatal rat $(n=10)$ was sampled. Isoflurane exposure increased cleaved caspase 3 as indicated in Figure 1A. To determine whether miR-497 is associated with isoflurane exposure, the expression level of miR-497 was detected using qRT-PCR. The result suggested that miR-497 was significantly upregulated nearly 2.7 -fold after isoflurane exposure compared to the control (Fig. 1B). These results may imply the involvement of miR-497 in isoflurane-induced neurotoxicity. The rest of the rats $(\mathrm{n}=10)$ were keeping feeding till P57. The 


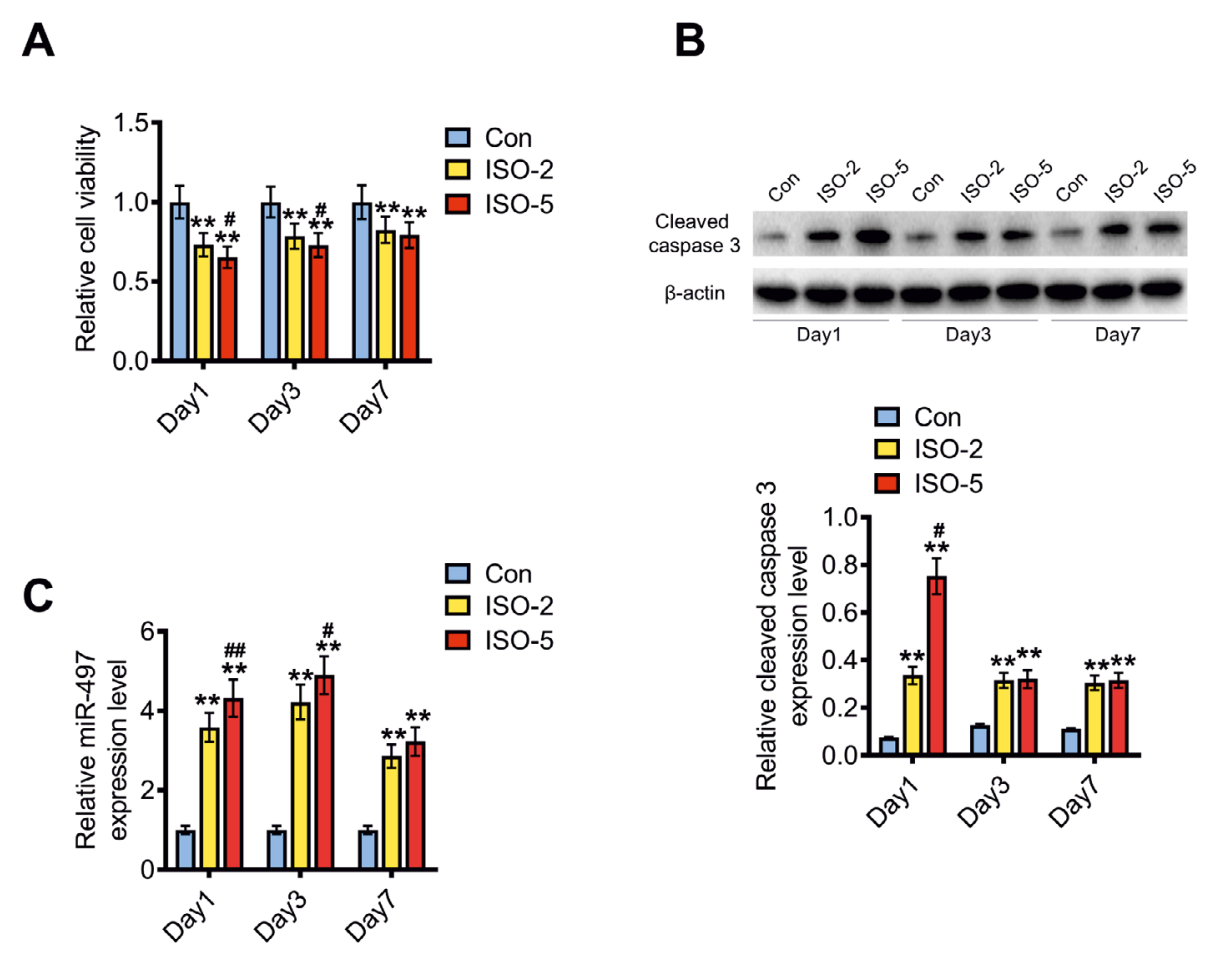

Figure 2. Isoflurane-induced neurotoxicity and miR-497 expression in primary cultures of hippocampal neurons. Neurons were cultured for 7 days and then exposed to two doses of isoflurane (ISO-2, $2 \%$ isoflurane; ISO-5, 5\% isoflurane) for $24 \mathrm{~h}$. (A) Cell viability (MTT test), (B) cleaved caspase 3 (western blot), and (C) miR-497 expression (RT-qPCR) were detected on the following days (D1, D3, D7). ${ }^{* *} p<0.01$, compared with control group, ${ }^{\#} p<0.05,{ }^{\# \#} p<0.01$, compared with ISO-2 group.

long-term neurocognitive impairment induced by isoflurane was evaluated by fear conditioning test and Morris water maze. As indicated in Figure 1C, rats exposed to isoflurane spent more time to find a submerged platform than the sham group. In the probe test, the number of platform crossing of rats exposed to isoflurane was reduced compared to the control group (Fig. 1D). In fear conditioning test, the freezing time of rats exposed to isoflurane decreased compared to that of the sham group (Fig. $1 \mathrm{E})$. These results implied that multiple exposures to isoflurane in newborns may lead to long-term cognitive impairment.

Upregulation of miR-497 induced by isoflurane was associated with apoptosis of neurons in primary culture Primary neurons cells isolated from rat embryos were exposed to $2 \%$ or $5 \%$ isoflurane for $24 \mathrm{~h}$, the cell viability was measured at 1,3, and 7 days after isoflurane exposure using MTT test. As indicated in Figure 2A, isoflurane exposure impaired neurons' viability, and a higher concentration of isoflurane exerted higher toxicity, moreover, the impaired viability of neurons was observed even 7 days after isoflurane exposure. The increased level of cleaved caspase 3 , which is a marker of cell apoptosis, was also observed after isoflurane exposure (Fig. 2B). The expression of miR-497 was significantly increased after isoflurane exposure, especially at 3 days after exposure (Fig. 2C). These results suggest isoflurane impairs neurons viability and induces apoptosis, and miR-497 may be involver in this process.

\section{MiR-497 inhibition mitigated isoflurane-induced apoptosis of hippocampal neurons in primary culture}

To examine the role of miR-497 in isoflurane-induced neurotoxicity, miR-497 inhibitor, or its negative control was transfected into primary neurons before isoflurane exposure. The transfection efficiency of miR-497 was confirmed by real-time PCR (Fig. 3A). After 7 days of $2 \%$ isoflurane exposure, the impaired cell viability of neurons cell induced by isoflurane was significantly alleviated with miR-497 inhibitor transfection (Fig. 3B). The miR-497 inhibition also decreased the level of cleaved caspase 3, which was dramatically induced by isoflurane exposure (Fig. 3C). These results suggested the protective role of miR-497 inhibition in neurotoxicity induced by isoflurane. 


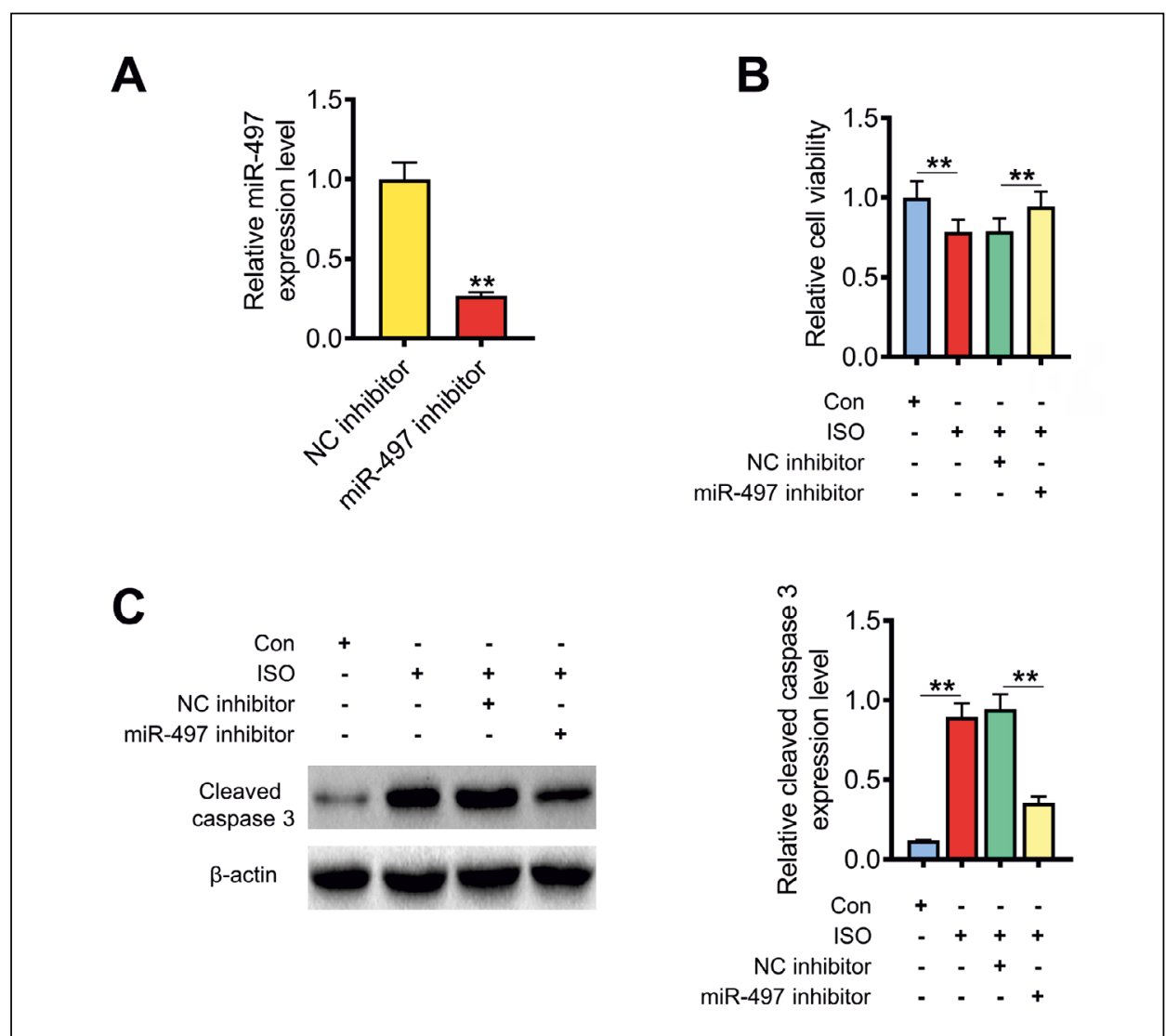

Figure 3. MiR-497 inhibitor ameliorated isoflurane-induced neurotoxicity in primary cultures of hippocampal neurons. A. Relative expression levels of miR-497 (RT-qPCR) in neuronal cells transfected with miR-497 inhibitor or negative control. B. Viability of cells transfected with miR-497 inhibitor or negative control followed by $2 \%$ isoflurane exposure for $24 \mathrm{~h}$. C. Expression of cleaved caspase 3 (western blot) in primary neuronal cell transfected with miR-497 inhibitor or negative control followed by $2 \%$ isoflurane exposure for 24 h. ${ }^{* *} p<0.01$.

\section{MiR-497 suppressed PLD1 expression by direct binding at the 3'UTR}

To explore the mechanism of miR-497 in isoflurane-induced neurotoxicity, the TargetScan (http:// www.targetscan.org/vert_72/) was used for predicting the target gene of miR-497, and PLD1 was predicted to be a potential target of miR-497 (Fig. 4A). Luciferase reporter assay was carried out to validate the direct interaction between miR-497 and PLD1. The 3'UTR region of PLD1 containing wide-type or mutant miR-497 binding sites was cloned into the pmirGLO vector. HEK-293T cells were co-transfected with reporter plasmids and miR-497 mimic or inhibitor or their negative control. As shown in Figure 4B, the ectopic expression of miR-497 significantly repressed luciferase activity, whereas miR-497 inhibition enhanced the luciferase activity. In contrast, 3 ' UTR mutations completely abrogated the effect of miR-497 on luciferase activity. Besides, the protein level of PLD1 was determined in neurons with miR497 overexpression or inhibition. Overexpression of miR-497 repressed protein levels of PLD1, while
miR-497 inhibition had the opposite effect (Fig. 4C). Furthermore, PLD1 was also detected in neurons treated with the indicated concentration of isoflurane and rat hippocampus after isoflurane anesthesia. As indicated in Fig. 4D, the protein level of PLD1 was dramatically suppressed after isoflurane exposure.

\section{Isoflurane exposure downregulated PLD1 expression and prompted neuronal cell death in rat pup's hippocampus}

We next detected the expression of PLD1 in rat pup's hippocampus after isoflurane exposure at days P7, P9 and P11. After isoflurane exposure, the protein abundance of PLD1 was reduced in hippocampus (Fig. 5A). Our IHC results demonstrated a dramatic reduction of PLD1-positive cells in hippocampus (Fig. 5B). The neuronal cell death in rat hippocampus was determined using TUNEL staining, and the results indicated an increasing portion of TUNEL-positive cells after isoflurane exposure (Fig. 5C). These results indicated that isoflurane exposure suppressed the expression of PLD1, and led to neurons cells death in rat pup's hippocampus. 


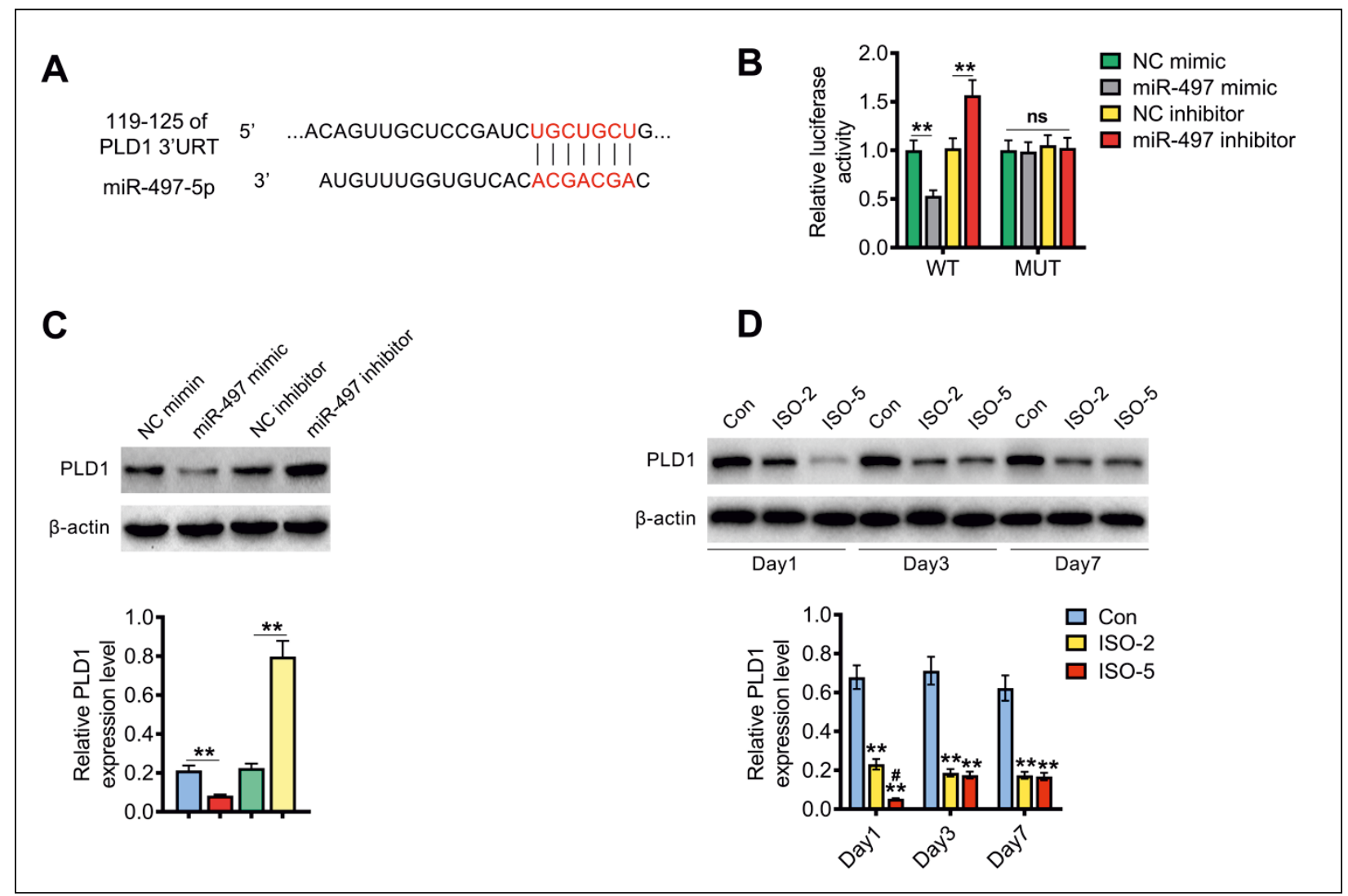

Figure 4. Phospholipase D1 (PLD1) is a novel target of miR-497. A. The predicted binding sites of miR-497 on 3'UTR of PLD1 based on TargetScan. B. Luciferase reporter assay was performed in HEK-293T cells to detect luciferase activity after co-transfection with miR-497 mimic, miR-497 inhibitor, or their negative control and dual-luciferase vector of 3'-UTR of PLD1 harboring the seed sequence of the miR-497 wild-type binding sites (WT) or mutant binding sites (MUT). C. PLD1 protein expression (western blot) in primary neuronal cells transfected with miR-497 mimic, miR-497 inhibitor, or their negative control for $48 \mathrm{~h} .{ }^{* *} p<0.01$, compared with NC mimic or NC inhibitor group. D. PLD1 protein expression (western blot) in primary neuronal cells subjected to $2 \%$ and $5 \%$ isoflurane for $24 \mathrm{~h}$ after the indicated days of culture. ${ }^{* *} p<0.01$, compared with control group, ${ }^{\#} p<0.05$, compared with ISO-2 group.

\section{Discussion}

In the present study, we showed that multiple isoflurane exposure of neonatal rats caused apoptosis of hippocampal neurons and led to long-term impairment of cognitive and learning functions. There are numerous reports which showed that single or multiple isoflurane exposure at neonatal period will lead to cognitive impairment. For instance, rats repeatedly exposed to isoflurane on $\mathrm{P} 7$ were found to have a long-term impaired of cognitive function, however, the cognitive impairments induced by a single exposure to isoflurane varied by gender [22]. The long-term impairment induced by neonatal isoflurane exposure perhaps largely results from neuronal apoptosis in the developing brain. As indicated in other study in which single isoflurane anesthesia of rats from 1 to 14 postnatal day, the highest level of apoptotic neuronal death was induced in neonatal rats at $\mathrm{P} 7$, the lowest neuronal apoptosis was seen in P14 rats. This finding could be explained by the fact that $\mathrm{P} 7$ rats are more vulnerable to be damaged since at this age the peak of synaptogenesis takes place [25]. Interestingly, some studies demonstrated that adult and aged rodents exposed to isoflurane also developed cognitive impairment [23, 26, 27]. Zhong et al. found that neonatal male mice exposed repeatedly to isoflurane exhibited dysregulated hippocampal H4K12 acetylation and decreased c-Fos expression, and administration of a histone deacetylase inhibitor could attenuate the impairment [28]. An another report revealed the possibility of the activation of hypoxia inducible factor- $1 \alpha$ (HIF- 1 $\alpha$ ) in isoflurane-induced neonatal neurotoxicity [29]. Additionally, TLR2/NF- $\kappa$ B pathway was proved to be involved in isoflurane-induced neurotoxicity [30]. Although great efforts have been done in elucidating the developmental neurotoxicity of isoflurane, the 




Figure 5. Isoflurane exposure downregulated PLD1 expression and prompted neuronal cell death in rat pup's hippocampus. After 3 independent isoflurane exposures at days P7, P9 and P11, the protein expression of PLD1 in rat pup's hippocampus (24 h after the last exposure) was examined by western blot (A) and immunohistochemistry (CA1) (B). C. Death of hippocampal neurons was evaluated using TUNEL assay in hippocampal CA1 after isoflurane exposure. Magnification for (B) and (C): $200 \times$.

underlying mechanisms have not been completely clarified so far.

The involvement of the crucial roles of miRNAs in various human diseases has been widely demonstrated. Besides, some recent studies also indicated that miRNAs may participate in neurotoxicity induced by isoflurane. For instance, miR-142-5p was found to be upregulated in neurological impairment caused by isoflurane in neonatal rats, whereas the inhibition of miR-142-5p exerted neuroprotective effects against isoflurane, reflected by a better behavior performance [31]. Moreover, isoflurane facilitated the expression of miR-140-5p in diabetic rat hippocampus, which led to the post-transcriptional suppression of sorting protein nexin 12 , and the depletion of sorting nexin 12 subsequently contributed to isoflurane neurotoxicity [32].

The main purpose of our study focuses on the role of miR-497 in isoflurane-induced neurons apoptosis and long-term cognitive impairment. Here, we showed that neonatal rats and cultured hippocampal neurons subjected to isoflurane displayed an obvious induction of miR-497. To clarify the role of miR-497 in isoflurane-induced neurotoxicity, miR-497 depletion using miR-497 inhibitor was conducted in cultured neurons and our results confirmed the neuroprotective effect of miR-497, as assessed by increased cell viability and decreased apoptosis. It was previously reported that the induction of miR-497 in neuronal cells exposed to ethanol and overexpression of miR-497 in the absence of ethanol also leads to neuronal cell apoptosis [21]. miR-497 also plays a role in oxygen-glucose depri- vation-induced neuronal cell death [20], implying the potential anti-apoptotic effect of miR-497 in the central nervous system.

Most importantly, our results further established PLD1 as a direct target of miR-497 in neuronal cells. PLD1 is a member of phospholipase D lipid-signaling enzyme superfamily and has been widely reported to function in various neuronal processes. The deficiency of PLD1 led in mice to defects in brain growth and cognitive function, moreover, upon stimulation the PLD-deficient mice released less acetylcholine than wild-type ones [33]. In addition, PLD1 was found to participate in BDNF-dependent nuclear recruitment of phospho-ERK1/2 and phospho-CREB, which may play role in neuronal development and induction of glutamatergic synapses, because it is known that BDNF plays a role in hippocampal long-term potentiations [34]. As synapses are responsible for information transmission, processing, and signal integration in the nervous system, PLD1 was found to promote the development of the dendritic spines by regulating $\mathrm{N}$-cadherin [35]. A similar conclusion was drawn in another report: genetic or pharmacological inhibition of PLD1 or its upstream RSK2 would lead to the impairment of neuronal growth factor-induced neurite outgrowth [36]. A recent study revealed that PLD1 activation attenuates coflin-p53 pro-apoptotic pathway induced by amyloid $\beta$, exerting its role in anti-apoptosis of neuronal cells [37]. In our present study, isoflurane anesthesia suppressed the expression of PLD1 both in neonatal rat hippocampus and 
primary neuronal cell cultures, which may be the potential mechanism of isoflurane-induced neurotoxicity. Moreover, PLD1 was validated as a novel target of miR-497 and was negatively regulated by miR-497. Hence, we inferred that isoflurane-induced the expression of miR-497 and consequently inhibited PLD1 to contribute to the apoptosis of neuronal cells. However, the effect of miR-497/PLD1 on cognitive impairment induced by isoflurane in neonatal rats need to be further investigated.

Taken together, our present research demonstrated that the involvement of miR-497 in isoflurane-induced neurotoxicity and long-term neurocognitive impairment, and clarified PLD1 as the downstream target of miR-497. This work may shed light on the underlying mechanisms of anesthetics-induced neurotoxicity and may contribute to developing novel therapeutic ways for isoflurane-related application.

\section{Acknowledgments}

Not applicable.

\section{Funding}

None.

\section{Availability of data and materials}

All data generated or analyzed during this study are included in this published article.

\section{Authors' contributions}

YYQ and FXZ designed the study, supervised the data collection and analyzed the data; JP and ZHZ interpreted the data and prepared the manuscript for publication; DWZ and MHH supervised the data collection, analyzed the data and reviewed the draft of the manuscript.

All authors have read and approved the manuscript.

\section{Ethics approval and consent to participate}

The animal use protocol listed below has been reviewed and approved by the Animal Ethical and Welfare Committee.

\section{Conflict of interests}

The authors declare that they have no competing interests, and all authors should confirm their accuracy.

\section{References}

1. Stratmann G, Sall JW, May LDV, et al. Isoflurane differentially affects neurogenesis and long-term neurocognitive function in 60-day-old and 7-day-old rats. Anesthesiology. 2009; 110(4): 834-848, doi: 10.1097/ALN.0b013e31819c463d, indexed in Pubmed: 19293705.

2. Flick RP, Katusic SK, Colligan RC, et al. Cognitive and behavioral outcomes after early exposure to anesthesia and surgery. Pediatrics. 2011; 128(5): e1053-e1061, doi: 10.1542/ peds.2011-0351, indexed in Pubmed: 21969289.

3. Sprung J, Flick RP, Wilder RT, et al. Anesthesia for cesarean delivery and learning disabilities in a population-based birth cohort. Anesthesiology. 2009; 111(2): 302-310, doi: 10.1097/ ALN.0b013e3181adf481, indexed in Pubmed: 19602960.

4. Lu LX, Yon JH, Carter LB, et al. General anesthesia activates BDNF-dependent neuroapoptosis in the developing rat brain. Apoptosis. 2006; 11(9): 1603-1615, doi: 10.1007/s10495-0068762-3, indexed in Pubmed: 16738805.

5. Creeley CE. From drug-induced developmental neuroapoptosis to pediatric anesthetic neurotoxicity-where are we now? Brain Sci. 2016; 6(3), doi: 10.3390/brainsci6030032, indexed in Pubmed: 27537919.

6. Cabrera OH, O'Connor SD, Swiney BS, et al. Caffeine combined with sedative/anesthetic drugs triggers widespread neuroapoptosis in a mouse model of prematurity. J Matern Fetal Neonatal Med. 2017; 30(22): 2734-2741, doi: 10.1080/14767058.2016.1261400, indexed in Pubmed: 27924651.

7. Stratmann G, May LDV, Sall JW, et al. Effect of hypercarbia and isoflurane on brain cell death and neurocognitive dysfunction in 7-day-old rats. Anesthesiology. 2009; 110(4): 849-861, doi: 10.1097/ALN.0b013e31819c7140, indexed in Pubmed: 19293696.

8. Yang S, Gu C, Mandeville ET, et al. Anesthesia and surgery impair blood-brain barrier and cognitive function in mice. Front Immunol. 2017; 8: 902, doi: 10.3389/fimmu.2017.00902, indexed in Pubmed: 28848542.

9. Yu D, Li L, Yuan W. Neonatal anesthetic neurotoxicity: Insight into the molecular mechanisms of long-term neurocognitive deficits. Biomed Pharmacother. 2017; 87: 196-199, doi: 10.1016/j.biopha.2016.12.062, indexed in Pubmed: 28056424.

10. Clausen NG, Hansen TG, Disma N. Anesthesia neurotoxicity in the developing brain: basic studies relevant for neonatal or perinatal medicine. Clin Perinatol. 2019; 46(4): 647-656, doi: 10.1016/j.clp.2019.08.002, indexed in Pubmed: 31653300.

11. Piscopo P, Lacorte E, Feligioni M, et al. MicroRNAs and mild cognitive impairment: A systematic review. Ageing Res Rev. 2019; 50: 131-141, doi: 10.1016/j.arr.2018.11.005, indexed in Pubmed: 30472218.

12. Sun E, Shi Y. MicroRNAs: Small molecules with big roles in neurodevelopment and diseases. Exp Neurol. 2015; 268 : 46-53, doi: 10.1016/j.expneurol.2014.08.005, indexed in Pubmed: 25128264 .

13. Issler $\mathrm{O}$, Chen A. Determining the role of microRNAs in psychiatric disorders. Nat Rev Neurosci. 2015; 16(4): 201-212, doi: 10.1038/nrn3879, indexed in Pubmed: 25790865.

14. Mai H, Fan W, Wang Y, et al. Intranasal administration of miR-146a agomir rescued the pathological process and cognitive impairment in an AD mouse model. Mol Ther Nucleic Acids. 2019; 18: 681-695, doi: 10.1016/j.omtn.2019.10.002, indexed in Pubmed: 31707205.

15. Goto G, Hori Y, Ishikawa M, et al. Changes in the gene expression levels of microRNAs in the rat hippocampus by sevoflurane and propofol anesthesia. Mol Med Rep. 2014; 9(5): 1715-1722, doi: 10.3892/mmr.2014.2038, indexed in Pubmed: 24626427.

16. Zhang $\mathrm{N}$, Ye $\mathrm{W}$, Wang $\mathrm{T}$, et al. Up-regulation of miR-106a targets LIMK1 and contributes to cognitive impairment induced by isoflurane anesthesia in mice. Genes Genomics. 2020; 42(4): 405-412, doi: 10.1007/s13258-019-00913-8, indexed in Pubmed: 31933141.

17. Yan $\mathrm{H}, \mathrm{Xu} \mathrm{T}, \mathrm{Zhao} \mathrm{H}$, et al. Isoflurane increases neuronal cell death vulnerability by downregulating miR-214. PLoS 
One. 2013; 8(2): e55276, doi: 10.1371/journal.pone.0055276, indexed in Pubmed: 23408966.

18. Lu X, Lv S, Mi Y, et al. Neuroprotective effect of miR-665 against sevoflurane anesthesia-induced cognitive dysfunction in rats through PI3K/Akt signaling pathway by targeting insulin-like growth factor 2. Am J Transl Res. 2017; 9(3): 1344-1356, indexed in Pubmed: 28386360.

19. Itesako T, Seki N, Yoshino H, et al. The microRNA expression signature of bladder cancer by deep sequencing: the functional significance of the miR-195/497 cluster. PLoS One. 2014; 9(2): e84311, doi: 10.1371/journal.pone.0084311, indexed in Pubmed: 24520312.

20. Yin KJ, Deng Z, Huang H, et al. miR-497 regulates neuronal death in mouse brain after transient focal cerebral ischemia. Neurobiol Dis. 2010; 38(1): 17-26, doi: 10.1016/j. nbd.2009.12.021, indexed in Pubmed: 20053374.

21. Yadav S, Pandey A, Shukla A, et al. miR-497 and miR-302b regulate ethanol-induced neuronal cell death through BCL2 protein and cyclin D2. J Biol Chem. 2011; 286(43): 3734737357, doi: 10.1074/jbc.M111.235531, indexed in Pubmed: 21878650

22. Murphy KL, McGaughy J, Croxson PL, et al. Long-term effects of neonatal single or multiple isoflurane exposures on spatial memory in rats. Front Neurol. 2013; 4(1): 87-95, doi: 10.3389/fneur.2013.00087, indexed in Pubmed: 23847588.

23. Lin $\mathrm{D}, \mathrm{Zuo} \mathrm{Z}$. Isoflurane induces hippocampal cell injury and cognitive impairments in adult rats. Neuropharmacology. 2011; 61(8): 1354-1359, doi: 10.1016/j.neuropharm.2011.08.011, indexed in Pubmed: 21864548.

24. Yan J, Huang Y, Lu Yi, et al. Repeated administration of ketamine can induce hippocampal neurodegeneration and long-term cognitive impairment via the ROS/HIF-1 $\alpha$ pathway in developing rats. Cell Physiol Biochem. 2014; 33(6): 1715-1732, doi: 10.1159/000362953, indexed in Pubmed: 24923288.

25. Yon JH, Daniel-Johnson J, Carter LB, et al. Anesthesia induces neuronal cell death in the developing rat brain via the intrinsic and extrinsic apoptotic pathways. Neuroscience. 2005; 135(3): 815-827, doi: 10.1016/j.neuroscience.2005.03.064, indexed in Pubmed: 16154281.

26. Li XM, Zhou MT, Wang XM, et al. Resveratrol pretreatment attenuates the isoflurane-induced cognitive impairment through its anti-inflammation and -apoptosis actions in aged mice. J Mol Neurosci. 2014; 52(2): 286-293, doi: 10.1007/ s12031-013-0141-2, indexed in Pubmed: 24126892.

27. Kong F, Chen S, Cheng Y, et al. Minocycline attenuates cognitive impairment induced by isoflurane anesthesia in aged rats. PLoS One. 2013; 8(4): e61385, doi: 10.1371/journal. pone.0061385, indexed in Pubmed: 23613842.

28. Zhong T, Guo Q, Zou W, et al. Neonatal isoflurane exposure induces neurocognitive impairment and abnormal hippocampal histone acetylation in mice. PLoS One. 2015; 10(4): e0125815, doi: 10.1371/journal.pone.0125815, indexed in Pubmed: 25928815.

29. Chai D, Jiang H, Li Q. Isoflurane neurotoxicity involves activation of hypoxia inducible factor- $1 \alpha$ via intracellular calcium in neonatal rodents. Brain Res. 2016; 1653: 39-50, doi: 10.1016/j.brainres.2016.10.014, indexed in Pubmed: 27769790.

30. Pang X, Zhang P, Zhou Y, et al. Dexmedetomidine pretreatment attenuates isoflurane-induced neurotoxicity via inhibiting the TLR2/NF- $\kappa$ B signaling pathway in neonatal rats. Exp Mol Pathol. 2020; 112: 104328, doi: 10.1016/j.yexmp.2019.104328, indexed in Pubmed: 31678237.

31. Xie C, Wang H, Zhang Yu, et al. Neuroprotective effects of miR-142-5p downregulation against isoflurane-induced neurological impairment. Diagn Pathol. 2020; 15(1): 70, doi: 10.1186/s13000-020-00978-0, indexed in Pubmed: 32505188.

32. Fan D, Yang S, Han Y, et al. Isoflurane-induced expression of miR-140-5p aggravates neurotoxicity in diabetic rats by targeting SNX12. J Toxicol Sci. 2020; 45(2): 69-76, doi: 10.2131/ jts.45.69, indexed in Pubmed: 32062618.

33. Burkhardt U, Stegner D, Hattingen E, et al. Impaired brain development and reduced cognitive function in phospholipase D-deficient mice. Neurosci Lett. 2014; 572: 48-52, doi: 10.1016/j.neulet.2014.04.052, indexed in Pubmed: 24813107.

34. Ammar MR, Thahouly T, Hanauer A, et al. PLD1 participates in BDNF-induced signalling in cortical neurons. Sci Rep. 2015; 5: 14778, doi: 10.1038/srep14778, indexed in Pubmed: 26437780.

35. Luo LD, Li G, Wang Y. PLD1 promotes dendritic spine development by inhibiting ADAM10-mediated N-cadherin cleavage. Scientific Reports. 2017; 7(1), doi: 10.1038/s41598017-06121-2, indexed in Pubmed: 28729535.

36. Ammar MR, Humeau Y, Hanauer A, et al. The Coffin-Lowry syndrome-associated protein RSK2 regulates neurite outgrowth through phosphorylation of phospholipase D1 (PLD1) and synthesis of phosphatidic acid. J Neurosci. 2013; 33(50): 19470-19479, doi: 10.1523/JNEUROSCI.2283-13.2013, indexed in Pubmed: 24336713.

37. Liu T, Wang F, LePochat $P$, et al. Cofilin-mediated Neuronal Apoptosis via p53 Translocation and PLD1 Regulation. Sci Rep. 2017; 7(1): 11532, doi: 10.1038/s41598-017-09996-3, indexed in Pubmed: 28912445.

Submitted: 2 November, 2020

Accepted after reviews: 27 April, 2021 Available as AoP: 18 May, 2021 